\title{
Greater Adherence to Dietary Guidelines were Associated with Lower Risk of Cardiovascular Diseases in Chinese Patients with Type 2 Diabetes: A Case-Control Study
}

\section{Shang-Ling Wu}

The First Affiliated Hospital of Sun Yat-sen University

Yan-Bin Ye ( $\nabla$ yeyanbin@mail.sysu.edu.cn )

The First Affiliated Hospital of Sun Yat-sen University

\section{Long-Yun Peng}

The First Affiliated Hospital of Sun Yat-sen University

\section{Yu-Ming Chen}

Sun Yat-sen University

\section{Fang-Fang Zeng}

Jinan University

\section{Shu-Yu Zhuo}

The First Affiliated Hospital of Sun Yat-sen University

\section{Yan-Bing Li}

The First Affiliated Hospital of Chongqing Medical University

\section{Wei Lu}

The First Affiliated Hospital of Sun Yat-sen University

\section{Pei-Yan Chen}

The First Affiliated Hospital of Sun Yat-sen University

\section{Hai-Yan Mai}

The First Affiliated Hospital of Sun Yat-sen University

\section{Shi Fang}

The First Affiliated Hospital of Sun Yat-sen University

Yi Sui

The First Affiliated Hospital of Sun Yat-sen University

\section{Pei-Shan Ruan}

The First Affiliated Hospital of Sun Yat-sen University

\section{Research}


Keywords: dietary index, dietary pattern, cardiovascular disease, case-control study, type 2 diabetes, Chinese

Posted Date: November 5th, 2020

DOI: https://doi.org/10.21203/rs.3.rs-100628/v1

License: (1) This work is licensed under a Creative Commons Attribution 4.0 International License. Read Full License 


\section{Abstract}

Background The evidence regarding the impact of the healthy eating index on the risk of cardiovascular events among patients with type 2 diabetes (T2D) is limited. To examine the associations of adherence to the Chinese and American dietary guidelines and the risk of cardiovascular disease (CVD) among Chinese individuals with T2D.

Methods This 1:1 matched case-control study included 419 enrolled pairs hospital-based CVD cases and controls who were matched by age and sex, in Guangdong province, China, all of whom had T2D. A structured questionnaire and a 79-item food-frequency questionnaire was used to collect general information and dietary intake information. Diet quality scores were calculated according to the Chinese Healthy Eating Index (CHEI) and the Healthy Eating Index-2015 (HEI-2015).

Results After adjustment for various confounding factors, the higher diet quality scores on the $\mathrm{CHEl}$ and HEl-2015 showed a significant association with a lower risk of CVD. The odds ratios (95\% confidence interval) per 5-score increment were $0.88(0.83,0.92)$ in the $\mathrm{CHEl}$ and $0.80(0.74,0.88)$ in the $\mathrm{HEI}-2015$, respectively. In stratified analyses, the protective associations remained significant in the subgroups of sex, smoking status, tea-drinking, hypertension state, dyslipidemia state, BMI, and T2D duration, but not among the drinkers in CHEI.

Conclusion Greater adherence to the most recent Chinese or American dietary guidelines were associated with a lower risk of CVD incidents among Chinese patients with T2D.

\section{Introduction}

Type 2 diabetes (T2D) is a worrying global epidemic and is of particular concern in China [1]. The leading cause of death in T2D remains cardiovascular disease (CVD) and hyperglycemia is associated with increased cardiovascular risk [2]. Several studies have shown that the patients with T2D had a 2-to 4-fold greater risk of developing CVD than those of nondiabetics [3]. It is momentous to identify cost-effective strategies for the prevention of cardiovascular complications due to diabetes.

Apart from pharmacological treatment, dietary modification is a fundamental therapy for selfmanagement of diabetes [4]. Previous studies have consistently reported an inverse association between the risk of CVD events and the consumption of individual food items such as fruit (Aune et al., 2017), vegetables [6], whole grains [7], seafood [8], whereas unprocessed red/processed meat [9] and salt [10] are positively associated. By thinking about the multiple dietary exposures' complexity and interrelations, overall dietary pattern analysis, which involves series methods to assess diets comprehensively, might be more informative about the role of diet for the etiology of diet-related diseases than single food item analysis [11].

Among these methods, diet quality indices developed on the strength of healthy dietary recommendations and available evidence of the diseases are design to assess compliance with dietary 
guidelines [12]. Substantial indices have been developed to evaluate integral dietary quality according to various dietary guidelines, as indicated by e.g., the Healthy Eating Index (HEI), the Mediterranean Diet Quality Index (MDQI), the alternate Healthy Eating Index (aHEI), and The Dietary Guidelines Index (DGI) [13]. Many studies [14-16] have implied that higher overall diet scores significantly reduce the risk of cardiovascular events in general populations, but the evidence regarding the effect of diet quality indices following a diabetes diagnosis on the risk of subsequent incident of CVD is limited, particularly in the Chinese population. It is well known that the diet recommended for diabetic patients varies from that of general healthy populations, therefore it is necessary to explore the dietary patterns which would be a benefit for the prevention of the complications of diabetes mellitus.

In the current study, we thus investigated the associations between two diet quality indexes, the Chinese Healthy Eating Index (CHEI) [17] and the latest version of the HEI (HEl-2015) [18], which reflects the 2016 Dietary Guidelines for the Chinese population [19] and the 2015-2020 Dietary Guidelines for the American population [20], respectively, and the risk regarding CVD among patients with T2D participating in a 1:1 matched case-control study undertaken in South China. We hope to provide some additional information to the guideline development for T2D.

\section{Methods}

\subsection{Study Population}

This case-control study was conducted among patients with existing T2D at the Endocrinology Department, the Neurology Department, and the Cardiology Department of The First Affiliated Hospital of Sun Yat-sen University, in Guangdong Province, China, from March 2013 to September 2015. Participants with previously diagnosed T2D, aged between 30 to 85 years, natives of Guangdong Province, or have lived in Guangdong for at least five years, and with a history at least 2 years of T2D were considered eligible for inclusion in the study. T2D was defined based on American Diabetes Association criteria (fasting plasma glucose $>7.0 \mathrm{mmol} \mathrm{L}^{-1}$ or 2-h plasma glucose $>11.1 \mathrm{mmol} \mathrm{L}^{-1}$, or both) [21]; or medication treatment. We excluded participants with (1) confirmed type-1 diabetes, gestational diabetes mellitus ( $n=20)$; (2) previous history of cancer, hepatic disease, renal disease, autoimmune disorders, diabetic retinopathy, and congenital heart disease $(n=182)$; (3) physical disability and disturbance of consciousness $(n=24)$; (4) significant changes in dietary habits or routine activities over the previous year ( $n=185)$; (5) incomplete dietary assessment ( $\geq 10 \%$ missing values) or an implausible intake of total daily energy ( $<700$ or $>4200 \mathrm{kcal}$ per day for males, $<500$ or $>3500 \mathrm{kcal}$ per day for females $[n=$ $11]) ;$ and (6) refusal of participate in the study $(n=39)$.

To conduct a case-control study, only patients with a new (diagnosed within 2 weeks) diagnosis of CVD at a date later than the T2D diagnosis were included in the case population. CVD were defined as nonfatal acute myocardial infarction, hospitalized unstable angina, and nonfatal stroke. Nonfatal myocardial infarction [22] and hospitalized unstable [23] angina was diagnosed according to the China Society of Cardiology of Chinese Medical Association criteria, including typical symptoms, elevated 
cardiac enzyme levels, and electrocardiographic findings. Nonfatal stroke was ascertained based on the national criteria, according to evidence of neurological deficits with sudden or rapid onset which persisted for a minimum of 24 hours [24]. The control group included patients with T2D who never had a selfreported CVD incident, exhibited no symptoms of cardiac involvement, had normal EKG levels, and had negative exercise tests. Finally, 419 eligible cases and 419 T2D controls, with and without CVD, frequency-matched by sex and age ( \pm 5 years), were included in the current study. All participants provided written informed consent, and the research protocol was approved by the Ethics Committee of The First Affiliated Hospital of Sun Yat-sen University (no. [2017]019).

\subsection{Data Collection}

Apart from hospital documented data (e.g., clinical characteristics and clinical examinations) for cases, the same questionnaires were used to collect general and dietary factors information during the 12 months prior to diagnosis (for the cases) or interview (for the controls). All of the participants were blind to the study hypothesis. In this study, both cases and controls completed a structured questionnaire via a face-to-face interview by a well-trained dietitian. We collected information regarding (1) sociodemographic characteristics (e.g., age, sex, education level); (2) lifestyles (e.g., tobacco smoking, alcohol consumption, and tea-drinking); (3) habitual dietary consumption during the one year prior to diagnosis (for the cases) or interview (for the controls); (4) history of chronic diseases and medication use (e.g., hypertension, dyslipidemia, insulin use, oral hypoglycemic use); and (5) physical activity. Education was grouped into three levels: primary school or below, middle or high school, and college or above. Participants who had been smoking at least one cigarette per day or drinking alcohol once a week continuously for at least six months were defined as smokers or alcohol drinkers. Tea drinkers were defined as individuals who drank tea at least twice a week. Physical activity was calculated using a 19item questionnaire by calculating the products of the time spent on a variety of activities (e.g., work, transportation, housework, leisure sedentary activity, and physical exercise) with the mean metabolic equivalent (MET) for that activity [25]. Anthropometric data including weight $(\mathrm{kg})$ and height $(\mathrm{m})$ were ascertained using standard procedures and measuring equipment by trained personnel. Body mass index (BMl, $\mathrm{kg} / \mathrm{m}^{2}$ ) was also calculated. Participants whose mean systolic blood pressure $\geq 140 \mathrm{mmHg}$ and/or diastolic blood pressure $\geq 90 \mathrm{mmHg}$ and/or in whom anti-hypertensive drug use were defined as hypertension [26]. Individuals whose cholesterol level was $\geq 200 \mathrm{mg} / \mathrm{dL}$, a low-density lipoprotein cholesterol level of $\geq 130 \mathrm{mg} / \mathrm{dL}$, a high-density lipoprotein cholesterol level of $<40 \mathrm{mg} / \mathrm{dL}$, or triglyceride level of $\geq 160 \mathrm{mg} / \mathrm{dL}$ or who were taking lipid-lowering medications was defined as dyslipidemia according to the China Atherosclerosis Society guidelines [27].

\subsection{Dietary Assessment}

A validated 79-item semiquantitative, paper-based, food-frequency questionnaire (FFQ) was used to collect dietary information [28]. For each food item, there were five possible frequencies (i.e., never, per year, per month, per week, and per day) and two predefined amounts of food consumption (i.e., servings or portion sizes) were recorded according to the choice of the participants during the previous year. We provided photographs of generic foods and standard portion sizes to help the participants estimate the 
amount of food they had usually ingested. The selected choice for each food was then converted to grams per day. Daily food and nutrient intakes were transformed into standard portions according to the 2016 Dietary Guidelines for the Chinese and the 2013-2014 Food Patterns Equivalents Database, respectively $[17,29]$. Daily dietary intakes of nutrients and total energy were calculated based on the Chinese Food Composition Table, 2009 [30]. Accounting for the information of salt ingestion, the detailed intake of salt was not collected in the FFQ, and we thus appointed a score to reflect a participants' sodium consumption according to taste preference (i.e., very salty, salty, moderate, mild, very mild) ranging from 0 to 10 by 2.5 .

\subsubsection{Chinese Healthy Eating Index (CHEI)}

The CHEI [17] is the first instrument in China to assess the overall diet quality adherence to the updated Dietary Guidelines for Chinese (DGC-2016) [19]. The total score of the CHEI ranges from 0 to 100, with 100 being the perfect adherence and 0 being nonadherence. The CHEl score consists of 17 components including 13 adequacy and 5 moderation food groups. Total grains ( $0-5$ points), whole grains and mixed beans ( $0-5$ points), tubers ( $0-5$ points), total vegetables $(0-5$ points $)$, dark vegetables $(0-5$ points $)$, dairy ( $0-5$ points), soybeans ( $0-5$ points), fish and seafood ( $0-5$ points), poultry ( $0-5$ points), eggs $(0-5$ points), seeds and nuts ( $0-5$ points), and fruit ( $0-10$ points) were adequacy components, representing dietary elements that are encouraged, and higher intake reflect higher scores. Red meat ( $0-5$ points), cooking oils ( $0-10$ points), sodium ( $0-10$ points), added sugars ( $0-5$ points), and alcohol ( $0-5$ points) were moderation components, which are dietary elements that are recommended to be limited, and lower ingestion reflected higher scores. Intermediate intake of every component was calculated proportionally. A score is given to each component and a total CHEl score is generated by calculating each component score. Each component is adjusted for total energy using the density method (per kilocalorie), except for sugar (percentage of energy) and alcohol (absolute consumptions). Higher overall CHEl scores signify better alignment with the latest Dietary Guidelines for the Chinese, and the validity and reliability of the CHEl have been examined explicitly [31]. Details are listed in Supplemental Table S1.

\subsubsection{Healthy eating index (HEI)-2015}

The HEl-2015 score [18], ranging from 0-100 possible points, which was exploited by the United States Department of Agriculture (USDA), was created to evaluate adherence to the 2015-2020 Dietary Guidelines for Americans (2015-2020 DGA) [20] and consists of 13 components including 9 adequacy and 4 moderation components, that were scored based on energy-adjusted food and nutrient intakes. The 3 adequacy components (i.e., whole grains, dairy, and fatty acids) are worth 0 to 10 points each, with 10 for highest and 0 for the lowest consumption. The remaining 6 adequacy components (i.e., total fruits, whole fruits, total vegetables, greens and beans, total protein foods, and seafood and plant proteins) are worth 0 to 5 points each, with 5 for the highest and 0 for the lowest ingestion. The 4 moderation components (i.e., refined grains, sodium, added sugars, and saturated fats) are recommended to be limited, and the lowest consumption of these dietary elements was scored as 10 , while the highest scored as 0 . Scores are counted proportionately according to the consumption between the minimum and 
maximum standards. A higher score indicates greater adherence to DGA recommendations and a better diet quality [18], and the HEI-2015 has good validity and reliability for assessing the diet quality relative to the updated Dietary Guidelines for Americans [32]. Details are listed in Supplemental Table S1.

\subsection{Statistical analysis}

All analyses were performed for males and females combined, except for analyses stratified by sex. For group comparisons, t-tests were used for the continuous variables with a normal distribution; Wilcoxon signed ranks tests were used for the others with a skewed distribution; and Chi square tests were used for the categorical variables, where appropriate. The scores of each component of the CHEI and HEI-2015 were divided into four groups, classified according to the range of percentage of full scores ([individual score/full score] 100\%): 0\% (0 point), $0.1-49.9 \%, 50-99.9 \%$ and $100 \%$ (full points), with corresponding value of $0,(0-50 \%$ of maximum score), ( $50 \%$ of maximum score), and maximum score, respectively. For the full scores of 5 , the cutoffs were $0,0.1-2.4,2.5-4.9$, and 5.0 , and for the full scores of 10 , the cutoffs were $0,0.1-4.9,5-9.9$, and 10.0. CVD odds ratios (ORs) and 95\% confidence intervals (Cls) were estimated using conditional logistic regression models, and we served the scores as continuous variables (per 5-point increments). Crude OR was calculated in the univariate model; and multivariable model was adjusted for age, BMI, marital status, physical activity, education level, smoking status, alcohol consumption, tea-drinking status, hypertension status, dyslipidemia status, T2D duration, antidiabetic medication using status, and non-alcohol energy intake. We also repeated all analyses with unconditional logistic regression modeling because if perfect matching were not possible, undertaking a strict matched analysis would result in the loss of relevant information[33]. Stratified analyses were conducted by sex, BMI ( $<24 v s$. $\geq 24 \mathrm{~kg} / \mathrm{m}^{2}$ ), smoking status (yes or no), alcohol consumption (yes or no), tea-drinking (yes or no), hypertension (yes or no), dyslipidemia (yes or no), T2D duration ( $<5 v$. $\geq 5$ years) and to calculate the multiplicative interactions by including each interaction item. All undertaken analyses were conducted using SPSS 23.0 (IBM Corp., Armonk, NY, USA) and $p<0.05$ was considered statistically significant.

\section{Results}

\subsection{Characteristics of the participants}

The selected characteristics of the 419 pairs ( 235 male pairs and 184 female pairs) of CVD cases and matched controls are shown in Table 1. The mean age was 61.13 years in the cases and 62.08 years in the controls. Compared with controls, the cases had significantly lower mean CHEl scores $(65.34 \pm 9.48$ vs. $71.31 \pm 9.05)$ and $\mathrm{HEl}-2015$ scores $(54.03 \pm 6.09$ vs. $57.77 \pm 6.79)$. All $p$-values were $<0.05$. The cases had lower physical activity, education level, T2D duration, and a lower proportion of tea-drinking and antidiabetic medication use, but they had a higher BMI, and there was a higher proportion of hypertension than in that of the controls. The CHEl scores were significantly correlated with the HEI-2015 scores (Spearman's $r=0.724 ; p<0.001$ ). 
Table 1

Characteristics of the study participants.

\begin{tabular}{|c|c|c|c|}
\hline Variable & $\begin{array}{l}\text { Control } \\
(n=419)\end{array}$ & $\begin{array}{l}\text { Case } \\
(n=419)\end{array}$ & $\begin{array}{l}p- \\
\text { value }\end{array}$ \\
\hline Age $(y)$ & $62.1(9.5)$ & $62.1(9.7)$ & 0.940 \\
\hline Sex, n (\%) & & & - \\
\hline Female & 184(43.9) & 184(43.9) & \\
\hline Male & $235(56.1)$ & $235(56.1)$ & \\
\hline $\mathrm{BMI}\left(\mathrm{kg} / \mathrm{m}^{2}\right)$ & $23.79(3.47)$ & $24.37(3.26)$ & 0.013 \\
\hline Smoker (\%) & & & 0.282 \\
\hline Yes & $115(27.4)$ & 131(31.3) & \\
\hline No & $304(72.6)$ & 287(68.7) & \\
\hline Alcohol consumption, n (\%) & & & 0.582 \\
\hline Yes & $370(88.3)$ & $375(89.5)$ & \\
\hline No & $49(11.7)$ & $44(10.5)$ & \\
\hline Tea drinking, n (\%) & $220(52.5)$ & 188(44.9) & 0.027 \\
\hline Physical activity (MET-h/d) & $26.05(24.38,28.46)$ & $25.56(23.93,27.65)$ & 0.015 \\
\hline Marital status, married, n (\%) & 401(95.7) & $396(94.5)$ & 0.423 \\
\hline Education level, n (\%) & & & 0.002 \\
\hline$<$ Middle school & $148(35.4)$ & $163(39.4)$ & \\
\hline Middle/High school & $102(24.4)$ & 132(31.9) & \\
\hline$\geq$ College & $168(40.2)$ & $119(28.7)$ & \\
\hline Hypertension, n (\%) & $206(49.5)$ & $313(75.2)$ & $\hat{0.001}$ \\
\hline Dyslipidemia, n (\%) & $230(57.1)$ & $234(61.7)$ & 0.184 \\
\hline
\end{tabular}

Abbreviations: BMI, body mass index; MET-h/day, Metabolic equivalent hours per day; $\mathrm{CHEl}$, Chinese Healthy Eating Index; HEl-2015, Healthy Eating Index 2015.

a Values are mean SD or median $\left(\mathrm{P}_{25}, \mathrm{P}_{75}\right)$, where appropriate.

b Total energy intake was dietary energy except for alcohol.

Statistically significant results are bolded. 


\begin{tabular}{|c|c|c|c|}
\hline Variable & $\begin{array}{l}\text { Control } \\
(n=419)\end{array}$ & $\begin{array}{l}\text { Case } \\
(n=419)\end{array}$ & $\begin{array}{l}p- \\
\text { value }\end{array}$ \\
\hline T2D duration (y) & $8.96(6.74)$ & $7.1(6.21)$ & $\hat{0} .001$ \\
\hline $\begin{array}{l}\text { Antidiabetic medication use, } \mathrm{n} \\
(\%)\end{array}$ & 408(99.5) & $375(97.9)$ & 0.043 \\
\hline CHEI & $71.31(9.05)$ & $65.34(9.48)$ & $\dot{\delta} 001$ \\
\hline HEI-2015 & $57.77(6.79)$ & $54.03(6.09)$ & $\dot{0} 001$ \\
\hline Total Energy $(\mathrm{kcal} / \mathrm{d})^{\mathrm{b}}$ & $\begin{array}{l}\text { 1439.00(1241.70, } \\
1703.70)\end{array}$ & $\begin{array}{l}\text { 1393.40(1187.10, } \\
\text { 1696.15) }\end{array}$ & 0.160 \\
\hline \multicolumn{4}{|c|}{$\begin{array}{l}\text { Abbreviations: BMI, body mass index; MET-h/day, Metabolic equivalent hours per day; CHEI, Chinese } \\
\text { Healthy Eating Index; HEI-2015, Healthy Eating Index } 2015 \text {. }\end{array}$} \\
\hline \multicolumn{4}{|c|}{ a Values are mean SD or median $\left(\mathrm{P}_{25}, \mathrm{P}_{75}\right)$, where appropriate. } \\
\hline \multicolumn{4}{|c|}{ b Total energy intake was dietary energy except for alcohol. } \\
\hline
\end{tabular}

\subsection{Participants in the Percentage distribution for each component}

The proportion in the percentage distribution of participants for the CHEI (A) and the HEl-2015 (B) component scores of each item are shown in Fig. 1. More than $50 \%$ of individuals achieved the recommendation (obtain the maximum points) of components for total grains, total vegetable, dark vegetable, fish and seafood, poultry, seeds and nuts, added sugars, and alcohol in the CHEl; and for total vegetable, greens and beans, total protein foods, seafood and plant proteins, added sugars, saturated fats in the HEI-2015. However, whole grains and mixed beans (65.5\%), dairy $(71.9 \%)$, soybeans $(59.8 \%)$ and fruits $(78.6 \%)$ in the $\mathrm{CHEl}$, and total fruits $(80.7 \%)$, whole fruits $(53.6 \%)$, whole grains $(87.9 \%)$, dairy $(91.4 \%)$ and refined grains $(94.9 \%)$ in the $\mathrm{HEl}-2015$ were components that had a relatively serious degree of deficiency intake (did not meet $50 \%$ of the recommendations) among the components. The score differences in the 10 components of the CHEl and 5 components of the HEI-2015 between cases and controls are all significant (all $p<0.05$ ). The control group scored better than the case group in food groups of whole grains and mix beans, tubers, total vegetables, dark vegetables, dairy, soybeans, and eggs in the $\mathrm{CHEl}$, and food groups of total fruits, whole fruits, total vegetables, whole grains, and dairy in the HEI-2015.

\subsection{Total risk score and stratified analysis.}


Associations between the CHEl and HEl-2015 scores and CVD risk are shown in Table 2. In the univariate model, participants with lower CHEl and HEl-2015 scores had a significantly increased risk of CVD (both $p<0.05)$. In the multivariable model, the risk persisted, and the ORs $(95 \% \mathrm{Cls})$ were $0.88(0.83,0.92)$ and $0.80(0.74,0.88)$ for per 5-point increment of CHEI and HEI-2015, respectively. In a stratified analysis of the subjects, the protective associations between the CHEI and HEI-2015 scores and CVD risk did not materially change according to the subgroups of sex, smoking status, tea-drinking, hypertension state, dyslipidemia state, BMI, and T2D duration, but not among the alcohol drinkers in $\mathrm{CHEl}$. The analyses were repeated with unconditional logistic regression modeling and not substantially different from the conditional logistic regression modeling for all comparisons (data not shown). 
Table 2

ORs (95\% Cls) of CVD for per 5-point increment of CHEl and HEl-2015 stratified by selected factors.

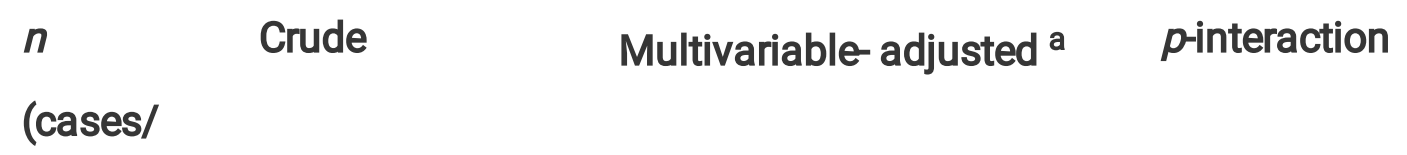

controls $) \quad$ OR $(95 \% \mathrm{Cl}) \quad$ OR $(95 \% \mathrm{Cl})$

\section{CHEI}

Total scored $\quad 419 / 419 \quad 0.86(0.82,0.90) \quad 0.88(0.83,0.92)$

$\begin{array}{ll}\text { Sex } & 0.088\end{array}$

$\begin{array}{llll}\text { Female } & 184 / 184 & 0.89(0.83,0.96) & 0.89(0.83,0.96) \\ \text { Male } & 235 / 235 & 0.82(0.77,0.87) & 0.85(0.79,0.92)\end{array}$

$\mathrm{BMl}, \mathrm{kg} / \mathrm{m}^{2}$

0.327

$\begin{array}{llll}\geq 24 & 198 / 184 & 0.89(0.82,0.95) & 0.91(0.84,0.98) \\ <24 & 221 / 235 & 0.84(0.79,0.89) & 0.86(0.80,0.92)\end{array}$

Smoker

0.541

\begin{tabular}{llll} 
Yes & $131 / 115$ & $0.83(0.76,0.90)$ & $0.87(0.79,0.96)$ \\
\hline No & $287 / 304$ & $0.87(0.82,0.92)$ & $0.88(0.83,0.94)$
\end{tabular}

Alcohol consumption

0.513

\begin{tabular}{llll} 
Yes & $44 / 49$ & $0.89(0.74,1.07)$ & $0.94(0.76,1.16)$ \\
\hline No & $375 / 370$ & $0.85(0.81,0.90)$ & $0.87(0.83,0.92)$
\end{tabular}

Tea-drinking

0.471

Yes $\quad 188 / 220 \quad 0.84(0.78,0.90) \quad 0.86(0.80,0.93)$

No $\quad 231 / 199 \quad 0.87(0.82,0.93) \quad 0.89(0.82,0.95)$

Hypertension

0.110

$\begin{array}{llll}\text { Yes } & 313 / 206 & 0.90(0.85,0.95) & 0.92(0.86,0.98)\end{array}$

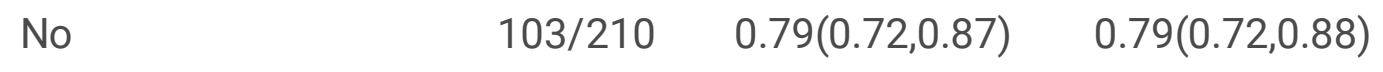

\section{Dyslipidemia}

0.646

Abbreviations: CHEI, Chinese Healthy Eating Index; HEI-2015, Healthy Eating Index 2015; ORs, Odds ratios, $\mathrm{Cl}$, Confidence Interval.

a Adjusted for age, body mass index, marital status, physical activity, education level, smoker status, alcohol consumption, tea-drinking status, hypertension status, dyslipidemia status, T2D duration, antidiabetic medication using status, and non-alcohol energy. 


\begin{tabular}{|c|c|c|c|c|}
\hline & $\begin{array}{l}n \\
\text { (cases/ }\end{array}$ & Crude & Multivariable- adjusted ${ }^{a}$ & p-interaction \\
\hline Yes & $234 / 230$ & $0.88(0.83,0.94)$ & $0.90(0.83,0.96)$ & \\
\hline No & $145 / 173$ & $0.81(0.75,0.88)$ & $0.83(0.75,0.92)$ & \\
\hline T2D duration, $y$ & & & & 0.922 \\
\hline$\geq 5$ & $227 / 269$ & $0.86(0.81,0.92)$ & $0.87(0.80,0.93)$ & \\
\hline$<5$ & $192 / 150$ & $0.86(0.80,0.92)$ & $0.87(0.81,0.94)$ & \\
\hline \multicolumn{5}{|l|}{ HEl-2015 } \\
\hline Total scored & $419 / 419$ & $0.79(0.73,0.86)$ & $0.80(0.74,0.88)$ & \\
\hline Sex & & & & 0.107 \\
\hline Female & $184 / 184$ & $0.83(0.74,0.94)$ & $0.87(0.76,0.98)$ & \\
\hline Male & $235 / 235$ & $0.72(0.64,0.81)$ & $0.76(0.67,0.86)$ & \\
\hline $\mathrm{BMl}, \mathrm{kg} / \mathrm{m}^{2}$ & & & & 0.116 \\
\hline$\geq 24$ & $198 / 184$ & $0.84(0.74,0.94)$ & $0.86(0.76,0.98)$ & \\
\hline$<24$ & $221 / 235$ & $0.76(0.68,0.84)$ & $0.76(0.67,0.86)$ & \\
\hline Smoker & & & & 0.465 \\
\hline Yes & $131 / 115$ & $0.73(0.62,0.86)$ & $0.77(0.65,0.92)$ & \\
\hline No & $287 / 304$ & $0.81(0.73,0.89)$ & $0.82(0.74,0.91)$ & \\
\hline Alcohol consumption & & & & 0.976 \\
\hline Yes & $44 / 49$ & $0.74(0.55,1.00)$ & $0.71(0.49,1.04)$ & \\
\hline No & $375 / 370$ & $0.79(0.73,0.86)$ & $0.81(0.74,0.88)$ & \\
\hline Tea-drinking & & & & 0.610 \\
\hline Yes & $188 / 220$ & $0.77(0.69,0.87)$ & $0.80(0.70,0.91)$ & \\
\hline No & $231 / 199$ & $0.80(0.72,0.89)$ & $0.81(0.72,0.91)$ & \\
\hline Hypertension & & & & 0.569 \\
\hline
\end{tabular}

Abbreviations: CHEl, Chinese Healthy Eating Index; HEI-2015, Healthy Eating Index 2015; ORs, Odds ratios, $\mathrm{Cl}$, Confidence Interval.

a Adjusted for age, body mass index, marital status, physical activity, education level, smoker status, alcohol consumption, tea-drinking status, hypertension status, dyslipidemia status, T2D duration, antidiabetic medication using status, and non-alcohol energy. 


\begin{tabular}{|c|c|c|c|c|}
\hline & $\begin{array}{l}n \\
\text { (cases/ }\end{array}$ & Crude & Multivariable-adjusted a & p-interaction \\
\hline Yes & $313 / 206$ & $0.86(0.78,0.95)$ & $0.88(0.79,0.98)$ & \\
\hline No & $103 / 210$ & $0.71(0.61,0.84)$ & $0.71(0.60,0.84)$ & \\
\hline Dyslipidemia & & & & 0.615 \\
\hline Yes & $234 / 230$ & $0.83(0.75,0.93)$ & $0.84(0.75,0.94)$ & \\
\hline No & $145 / 173$ & $0.75(0.65,0.86)$ & $0.76(0.65,0.89)$ & \\
\hline T2D duration, $y$ & & & & 0.267 \\
\hline$\geq 5$ & $227 / 269$ & $0.83(0.75,0.93)$ & $0.84(0.74,0.95)$ & \\
\hline$<5$ & $192 / 150$ & $0.76(0.67,0.85)$ & $0.76(0.67,0.86)$ & \\
\hline \multicolumn{5}{|c|}{$\begin{array}{l}\text { Abbreviations: CHEl, Chinese Healthy Eating Index; HEI-2015, Healthy Eating Index 2015; ORs, Odds } \\
\text { ratios, Cl, Confidence Interval. }\end{array}$} \\
\hline \multicolumn{5}{|c|}{$\begin{array}{l}\text { a Adjusted for age, body mass index, marital status, physical activity, education level, smoker status, } \\
\text { alcohol consumption, tea-drinking status, hypertension status, dyslipidemia status, T2D duration, } \\
\text { antidiabetic medication using status, and non-alcohol energy. }\end{array}$} \\
\hline
\end{tabular}

\subsection{Association of each component score with CVD}

Figure 2 presents the ORs and 95\% Cls of each component score of CHEI (A) and HEl-2015 (B) for CVD risk between the case and control groups. Compared with the case group, lower risk of CVD was associated with higher scores for the following components: whole grains and mixed beans (OR, 0.92; $95 \% \mathrm{Cl} 0.87,0.97)$, total vegetables $(\mathrm{OR}, 0.86 ; 95 \% \mathrm{Cl} 0.77,0.97)$, dark vegetables $(\mathrm{OR}, 0.89 ; 95 \% \mathrm{Cl} 0.80$, $0.98)$, dairy (OR, $0.91 ; 95 \% \mathrm{Cl} 0.86,0.96)$, eggs (OR, $0.86 ; 95 \% \mathrm{Cl} 0.92,0.98)$, fruit (OR, $0.94 ; 95 \% \mathrm{Cl} 0.90$, 0.97 ) and sodium (OR, $0.94 ; 95 \% \mathrm{Cl} 0.89,0.99)$ in the $\mathrm{CHEl}$; total fruits(OR, $0.87 ; 95 \% \mathrm{Cl} 0.81,0.95)$, whole fruits (OR, 0.90; $95 \% \mathrm{Cl} 0.85,0.96)$, whole grains (OR, 0.95; $95 \% \mathrm{Cl} 0.91,0.98)$, dairy (OR, $0.91 ; 95 \% \mathrm{Cl} 0.86$, $0.97)$, and sodium $(\mathrm{OR}, 0.94 ; 95 \% \mathrm{Cl} 0.89,0.99)$ in the HEI-2015.

\section{Discussion}

In this 1:1 case-control study with 419 pairs hospital-based CVD cases and T2D controls conducted in South China, we found a higher score between the CHEI or HEI-2015 after diabetes diagnosis, reflecting greater alignment with the latest and authoritative dietary guidelines for the Chinese or the Americans, and had strong favorable associations with lower risk of cardiovascular outcomes among Chinese adults with diabetes. 
Several studies and meta-analyses [14-16, 34, 35] have consistently shown that high diet quality, as assessed by HEI, AHEI, DASH, and Med score is inversely associated with the risk of CVD incidence in the general population. In the US Women's Health study which included 25,994 women (followed-up for 12 years), the consumption of a diet with a high baseline Med score may reduce the risk of cardiovascular events by one-fourth when comparing the upper (Med score 6-9) and lower (Med score $\leq$ 3) after adjustment for cardiovascular disease risk factors [16]. Similar findings were seen in 1867 middle-aged men from the Caerphilly Prospective Study, in which diets of the highest quality, as assessed by DASH and AHEI-2010 scores, resulted in a significant reduction in the incidence risk of CVD and stroke, but not coronary heart disease, with multivariable adjusted hazard ratios ranging from 0.61 to 0.81 in the highest (vs. lowest) tertiles [14]. Data from the prospective Singapore Chinese Health study showed that the AHEI-2010, which are strongly correlated with the HEl-2010, had an inverse association with the risk of fatal and nonfatal acute myocardial infarction among women and men ( $p$ trend, 0.009 and 0.002 , respectively) [15]. However, significant protective associations have only been shown between nut consumption [36] or overall healthy lifestyle [37] and the risk of CVD among diabetes patients in previous studies. Few studies have examined the risk of CVD regarding national dietary guidelines, particularly among persons with diabetes mellitus. It is unknown how to eat properly to prevent cardiovascular events in diabetic patients who are at high-risk of CVD. The finding of this study is in accordance with observational studies $[38,39]$ conducted in individuals with diabetes, showing a significant protective effect of intermediate cardiovascular risk factors profile, as indicated by e.g., fasting blood glucose, triglycerides, and systolic blood pressure, in patients who adhered mostly to the HEl-2010 (Daneshzad et al., 2019) or Mediterranean diet [39], yet an absence of data on CVD incident outcomes in these studies does not allow comparisons with our results. In general, our detections and existing evidence imply that aligning with a healthy dietary pattern after a diabetes diagnosis can momentously contribute to the prevention of cardiovascular complications among patients with T2D.

We also explored the relationship between each food group component scores for participants of CHEI and $\mathrm{HEI}-2015$ and the achievement of treatment targets for participants' risks. The beneficial effects of high-quality dietary scores may reflect the synergistic effects of diverse foods characterized by a higher intake of vegetables, fruit, whole grains, soybeans and dairy, and a moderate intake of cooking oils, sodium and red meat. Research has shown that whole grains and vegetables are primary sources of dietary fiber conducive to furthering hyperglycemia and improving dyslipidemia owing to the low glycemic index and anticholesterolemic actions [40]. Furthermore, micronutrients including minerals, vitamins, and phytochemicals are rich sources of these foods, all of which include insulin-sensitizing properties, are anti-inflammatory, reduce hypercoagulability, and regulate metabolic and antioxidant pathways to improve macro- and micro-vascular status [41]. The healthy dietary pattern mainly lies in its combined effect among all types of foods and nutrients instead of on any single component. Nonetheless, because our study was an observational study, this association should be interpreted with caution, as future biological mechanical research and possible interventional studies are needed to further illustrate potential mechanisms in the prevention of cardiovascular events among patients with T2D. 
In alcohol consumption-stratified analyses, the favorable association between $\mathrm{CHEl}$ and cardiovascular events remained significant in non-drinkers but not in drinkers. Taking into consideration the number of participants who drank alcohol, this may be just due to a low statistical power in this subgroup. In addition, the interaction with alcohol consumption was not statistically significant, which does not speak for a different association among drinkers and non-drinkers. Additionally, we further assessed the CHEI score after removing alcohol consumption from the categories, with the results being similar.

To our knowledge, our study assesses T2D patients' CVD risk associated with adherence to DGC-2016 and/or 2015-2020 DGA for the first time in a case-control design. Moreover, we only included newly diagnosed CVD patients with comparable age and sex to minimize recall bias. Furthermore, we excluded the participants with substantial changes in diet during the prior one year to ensure the representativeness of the habitual diet before diagnosis or interview. Additionally, multiple potential covariates, including explicit risk factors of CVD (i.e. hypertension status, dyslipidemia status, antidiabetic medication use) were included in the analyses for reducing residual confounding. Information bias was also further minimized because the participants were blinded to the hypothesis.

Nevertheless, several limitations of the study should be acknowledged. First, reverse causality could not be fully ruled out in the case-control design because the dietary intake information was assessed after the diagnosis of CVD. To minimize this possibility, we recruited only new cases (diagnosed within 2 weeks) into our study and collected the cases' diet information using the FFQ from the past twelve months prior to diagnosis. Second, although the FFQs used in our study were validated and implemented during face-to-face interviews by well-trained dietitians, dietary measurement errors are inevitable [42]. Third, the outcomes for the sodium component should be interpreted with caution because discretionary salt being used in cooking was not accurately captured in our FFQs. As with previous epidemiological studies, it is crude and likely to underestimate the ingestion of dietary sodium consumption assessment by using an FFQ. Finally, prior dietary indexes were derived based on current learning, and the CHEl and HEI-2015, originally directed toward general populations, rather than T2D, to prevent chronic disease.

\section{Conclusion}

The findings from this case-control study suggest that higher adherence to either Chinese or American dietary guidelines, as reflected in the $\mathrm{CHEl}$ and $\mathrm{HEI}-2015$, were similarly associated with a substantially lower risk of CVD among Chinese patients with T2D. Our results further support the current recommendation that patients with diabetes adopt a healthy dietary pattern may be a promising strategy for the prevention of CVD complications. Further studies, especially large prospective studies, are needed to replicate these findings.

\section{Abbreviations}

CVD: cardiovascular disease; T2D: type 2 diabetes; CHEl: the Chinese Healthy Eating Index; HEl: the Healthy Eating Index; aHEI: the alternate Healthy Eating Index; MDQI: the Mediterranean Diet Quality 
Index; DGI: The Dietary Guidelines Index; MET: metabolic equivalent; BMI: body mass index; FFQ: foodfrequency questionnaire; DGC: Dietary Guidelines for Chinese; MPED, MyPyramid Equivalents Database; USDA: the United States Department of Agriculture; DGA: Dietary Guidelines for Americans; OR: odds ratio; $\mathrm{Cl}$ : confidence interval.

\section{Declarations}

\section{Ethical Approval and Consent to participate}

All participants provided written informed consent, and the research protocol was approved by the Ethics Committee of The First Affiliated Hospital of Sun Yat-sen University (no. [2017]019)

\section{Consent for publication}

All authors of this paper have read and have approved the final submitted version.

\section{Availability of supporting data}

Participants' data cannot be shared because of the protection of data privacy.

\section{Competing interests}

The authors declare that they have no other conflicts of interest to disclose.

\section{Funding}

This work was supported by grants from the Youth Program of National Natural Science Foundation of China (Grant no. 81202197) and the Guangdong Natural Science Foundation (Grant no. 2015A030313011).

\section{Authors' contributions}

The authors' contributions are as follows: Yan-Bin Ye. and Yu-Ming Chen conceived and designed the study; Long-Yun Peng, Shu-Yu Zhuo, Yan-Bing Li, Wei Lu, Pei-Yan Chen, Hai-Yan Mai, Shi Fang, Yi Sui and Pei-Shan Ruan carried out the study; Shang-Ling Wu analyzed the data, wrote the paper., Shang-Ling Wu, Yan-Bin Yan, and Fang-Fang Zeng revised the final paper; all the authors read and approved the final version of the manuscript.

\section{Acknowledgements}

The authors thank the patients who agreed to participate in this study and complete the survey.

\section{References}


1. Wang L, Gao P, Zhang M, Huang Z, Zhang D, Deng Q, Li Y, Zhao Z, Qin X, Jin D et al: Prevalence and Ethnic Pattern of Diabetes and Prediabetes in China in 2013. JAMA 2017, 317(24):2515-2523.

2. 10. Cardiovascular Disease and Risk Management: Standards of Medical Care in Diabetes-2019. DIABETES CARE 2019, 42(Suppl 1):S103-S123.

3. Haffner SJ, Cassells H: Hyperglycemia as a cardiovascular risk factor. AM J MED 2003, 115 Suppl 8A:6S-11S.

4. 5. Lifestyle Management: Standards of Medical Care in Diabetes-2019. DIABETES CARE 2019, 42(Suppl 1):S46-S60.

5. Aune D, Giovannucci E, Boffetta P, Fadnes LT, Keum N, Norat T, Greenwood DC, Riboli E, Vatten LJ, Tonstad S: Fruit and vegetable intake and the risk of cardiovascular disease, total cancer and allcause mortality-a systematic review and dose-response meta-analysis of prospective studies. INT J EPIDEMIOL 2017, 46(3):1029-1056.

6. Zhan J, Liu YJ, Cai LB, Xu FR, Xie T, He QQ: Fruit and vegetable consumption and risk of cardiovascular disease: A meta-analysis of prospective cohort studies. Crit Rev Food Sci Nutr 2017, 57(8):1650-1663.

7. Mellen PB, Walsh TF, Herrington DM: Whole grain intake and cardiovascular disease: a metaanalysis. Nutr Metab Cardiovasc Dis 2008, 18(4):283-290.

8. Rimm EB, Appel LJ, Chiuve SE, Djoussé L, Engler MB, Kris-Etherton PM, Mozaffarian D, Siscovick DS, Lichtenstein AH: Seafood Long-Chain n-3 Polyunsaturated Fatty Acids and Cardiovascular Disease: A Science Advisory From the American Heart Association. CIRCULATION 2018, 138(1):e35-e47.

9. Zhong VW, Van Horn L, Greenland P, Carnethon MR, Ning H, Wilkins JT, Lloyd-Jones DM, Allen NB: Associations of Processed Meat, Unprocessed Red Meat, Poultry, or Fish Intake With Incident Cardiovascular Disease and All-Cause Mortality. JAMA INTERN MED 2020.

10. Smyth A, O'Donnell M, Mente A, Yusuf S: Dietary sodium and cardiovascular disease. CURR HYPERTENS REP 2015, 17(6):559.

11. Hu FB: Dietary pattern analysis: a new direction in nutritional epidemiology. CURR OPIN LIPIDOL 2002, 13(1):3-9.

12. Trichopoulos D, Lagiou P: Dietary patterns and mortality. Br J Nutr 2001, 85(2):133-134.

13. Arvaniti F, Panagiotakos DB: Healthy indexes in public health practice and research: a review. Crit Rev Food Sci Nutr 2008, 48(4):317-327.

14. Mertens E, Markey O, Geleijnse JM, Lovegrove JA, Givens DI: Adherence to a healthy diet in relation to cardiovascular incidence and risk markers: evidence from the Caerphilly Prospective Study. EUR J NUTR 2018, 57(3):1245-1258.

15. Neelakantan N, Naidoo N, Koh WP, Yuan JM, van Dam RM: The Alternative Healthy Eating Index Is Associated with a Lower Risk of Fatal and Nonfatal Acute Myocardial Infarction in a Chinese Adult Population. J NUTR 2016, 146(7):1379-1386. 
16. Ahmad S, Moorthy MV, Demler OV, Hu FB, Ridker PM, Chasman DI, Mora S: Assessment of Risk Factors and Biomarkers Associated With Risk of Cardiovascular Disease Among Women Consuming a Mediterranean Diet. JAMA Netw Open 2018, 1(8):e185708.

17. Yuan Y, Li F, Dong R, Chen J, He G, Li S, Chen B: The Development of a Chinese Healthy Eating Index and Its Application in the General Population. NUTRIENTS 2017, 9(9):977.

18. Krebs-Smith SM, Pannucci TE, Subar AF, Kirkpatrick SI, Lerman JL, Tooze JA, Wilson MM, Reedy J: Update of the Healthy Eating Index: HEl-2015. J ACAD NUTR DIET 2018, 118(9):1591-1602.

19. Wang SS, Lay S, Yu HN, Shen SR: Dietary Guidelines for Chinese Residents (2016): comments and comparisons. J Zhejiang Univ Sci B 2016, 17(9):649-656.

20. Agriculture UDO: Dietary guidelines for Americans 2015-2020. Eighth edition. In., vol. 2020.3; 2015.

21. Expert COTD: Report of the expert committee on the diagnosis and classification of diabetes mellitus. DIABETES CARE 2003, 26 Suppl 1:S5-S20.

22. [Guideline for diagnosis and treatment of patients with ST-elevation myocardial infarction]. Zhonghua Xin Xue Guan Bing Za Zhi 2010, 38(8):675-690.

23. [Guideline for diagnosis and treatment of patients with unstable angina and non-ST-segment elevation myocardial infarction]. Zhonghua Xin Xue Guan Bing Za Zhi 2007, 35(4):295-304.

24. Rao M: Chnia Guideline for cerebrovascular disease prevention and treatment. Beijing: People's Medical Publishing House; 2007.

25. Ainsworth BE, Haskell WL, Herrmann SD, Meckes N, Bassett DJ, Tudor-Locke C, Greer JL, Vezina J, Whitt-Glover MC, Leon AS: 2011 Compendium of Physical Activities: a second update of codes and MET values. Med Sci Sports Exerc 2011, 43(8):1575-1581.

26. Whitworth JA: $\mathbf{2 0 0 3}$ World Health Organization (WHO)/International Society of Hypertension (ISH) statement on management of hypertension. J HYPERTENS 2003, 21(11):1983-1992.

27. [2016 Chinese guideline for the management of dyslipidemia in adults]. Zhonghua Xin Xue Guan Bing Za Zhi 2016, 44(10):833-853.

28. Zhang $C$, Ho SC: Validity and reproducibility of a food frequency Questionnaire among Chinese women in Guangdong province. ASIA PAC J CLIN NUTR 2009, 18(2):240-250.

29. Bowman SA, Clemens JC, Friday JE, Lynch KL, Moshfegh AJ: Food Patterns Equivalents Database 2013-14: Methodology and User Guide. In., vol. 2020; 2017.

30. Yang Y, Wang G, Pan X: China Food Composition, 2nd ed. Beijing, China: Peking University Medical Press; 2009.

31. Yuan Y, Li F, Wu H, Wang Y, Chen J, He G, Li S, Chen B: Evaluation of the Validity and Reliability of the Chinese Healthy Eating Index. NUTRIENTS 2018, 10(2):114.

32. Reedy J, Lerman JL, Krebs-Smith SM, Kirkpatrick SI, Pannucci TE, Wilson MM, Subar AF, Kahle LL, Tooze JA: Evaluation of the Healthy Eating Index-2015. J ACAD NUTR DIET 2018, 118(9):1622-1633.

33. O'Donnell MJ, Xavier D, Liu L, Zhang H, Chin SL, Rao-Melacini P, Rangarajan S, Islam S, Pais P, McQueen MJ et al: Risk factors for ischaemic and intracerebral haemorrhagic stroke in 22 countries 
(the INTERSTROKE study): a case-control study. LANCET 2010, 376(9735):112-123.

34. Schwingshackl L, Bogensberger B, Hoffmann G: Diet Quality as Assessed by the Healthy Eating Index, Alternate Healthy Eating Index, Dietary Approaches to Stop Hypertension Score, and Health Outcomes: An Updated Systematic Review and Meta-Analysis of Cohort Studies. J ACAD NUTR DIET 2018, 118(1):74-100.

35. Becerra-Tomás N, Blanco MS, Viguiliouk E, Khan T, Kendall C, Kahleova H, Rahelić D, Sievenpiper JL, Salas-Salvadó J: Mediterranean diet, cardiovascular disease and mortality in diabetes: A systematic review and meta-analysis of prospective cohort studies and randomized clinical trials. Crit Rev Food Sci Nutr 2020, 60(7):1207-1227.

36. Liu G, Guasch-Ferré M, Hu Y, Li Y, Hu FB, Rimm EB, Manson JE, Rexrode KM, Sun Q: Nut Consumption in Relation to Cardiovascular Disease Incidence and Mortality Among Patients With Diabetes Mellitus. CIRC RES 2019, 124(6):920-929.

37. Liu G, Li Y, Hu Y, Zong G, Li S, Rimm EB, Hu FB, Manson JE, Rexrode KM, Shin HJ et al: Influence of Lifestyle on Incident Cardiovascular Disease and Mortality in Patients With Diabetes Mellitus. $J A M$ COLL CARDIOL 2018, 71(25):2867-2876.

38. Daneshzad E, Larijani B, Azadbakht L: Diet quality indices and cardiovascular diseases risk factors among diabetic women. J Sci Food Agric 2019, 99(13):5926-5933.

39. Vitale M, Masulli M, Calabrese I, Rivellese AA, Bonora E, Signorini S, Perriello G, Squatrito S, Buzzetti $\mathrm{R}$, Sartore G et al: Impact of a Mediterranean Dietary Pattern and Its Components on Cardiovascular Risk Factors, Glucose Control, and Body Weight in People with Type 2 Diabetes: A Real-Life Study. NUTRIENTS 2018, 10(8).

40. Babio N, Balanza R, Basulto J, Bulló M, Salas-Salvadó J: Dietary fibre: influence on body weight, glycemic control and plasma cholesterol profile. Nutr Hosp 2010, 25(3):327-340.

41. Archundia HM, Subhan FB, Chan CB: Dietary Patterns and Cardiovascular Disease Risk in People with Type 2 Diabetes. Curr Obes Rep 2017, 6(4):405-413.

42. Paeratakul S, Popkin BM, Kohlmeier L, Hertz-Picciotto I, Guo X, Edwards LJ: Measurement error in dietary data: implications for the epidemiologic study of the diet-disease relationship. EUR J CLIN NUTR1998, 52(10):722-727.

\section{Figures}




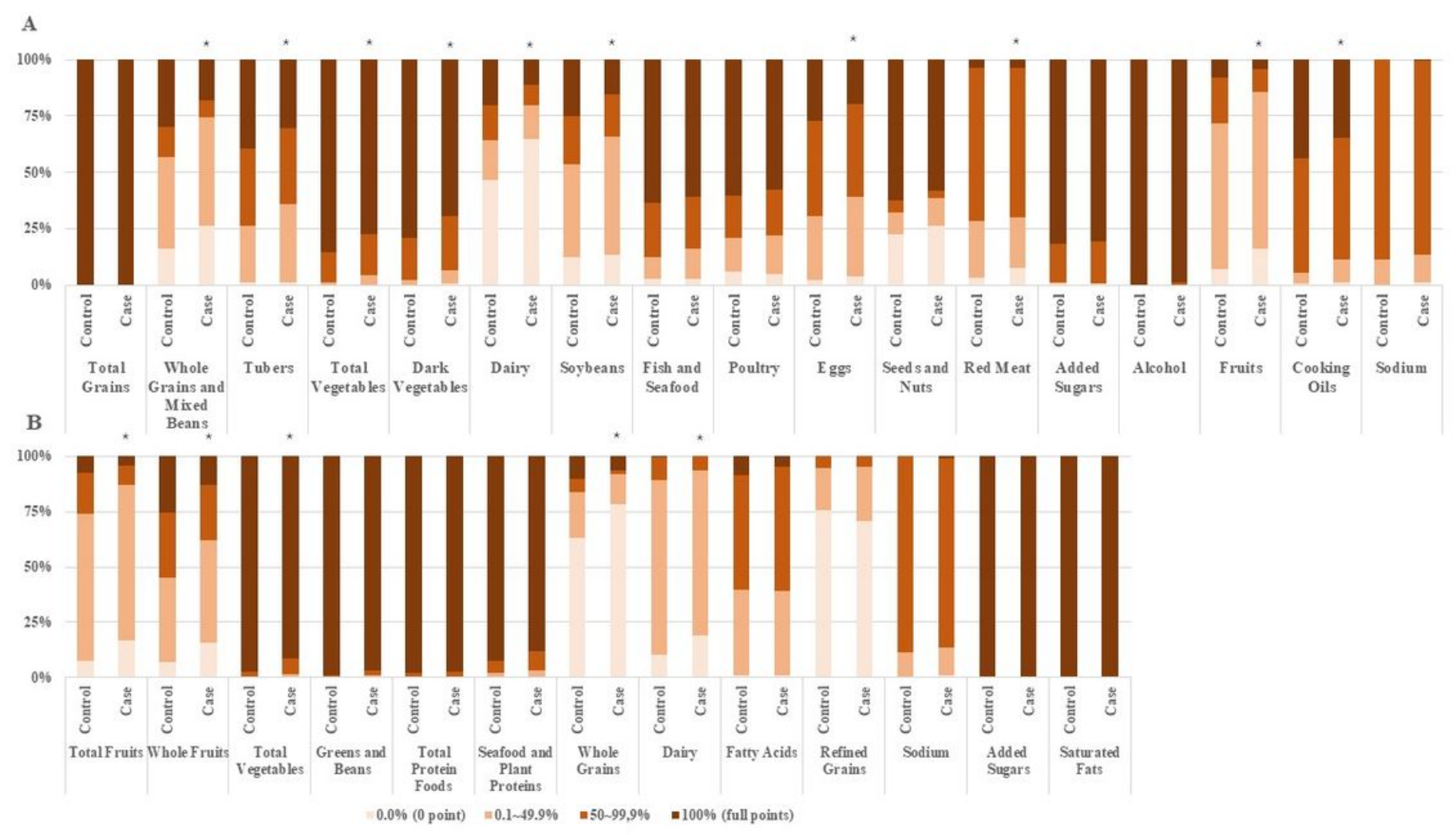

Figure 1

Comparison of the percentage distribution of the cases and controls according to the scores of each item for the CHEI (A) and the HEI-2015 (B). For fruits, sodium, and cooking oils in the CHEl and whole grains, dairy, fatty acids, refined grains, sodium, added sugars, and saturated fats in the HEI-2015, the cutoffs for the four groups are $0,0.1-4.9,5.0-9.9$, and 10.0. For the remaining components in the CHEl and the HEl2015 , the cutoffs for the four groups are $0,0.1-2.4,2.5-4.9$, and 5.0. *: $p<0.05$ (chi-square test). 


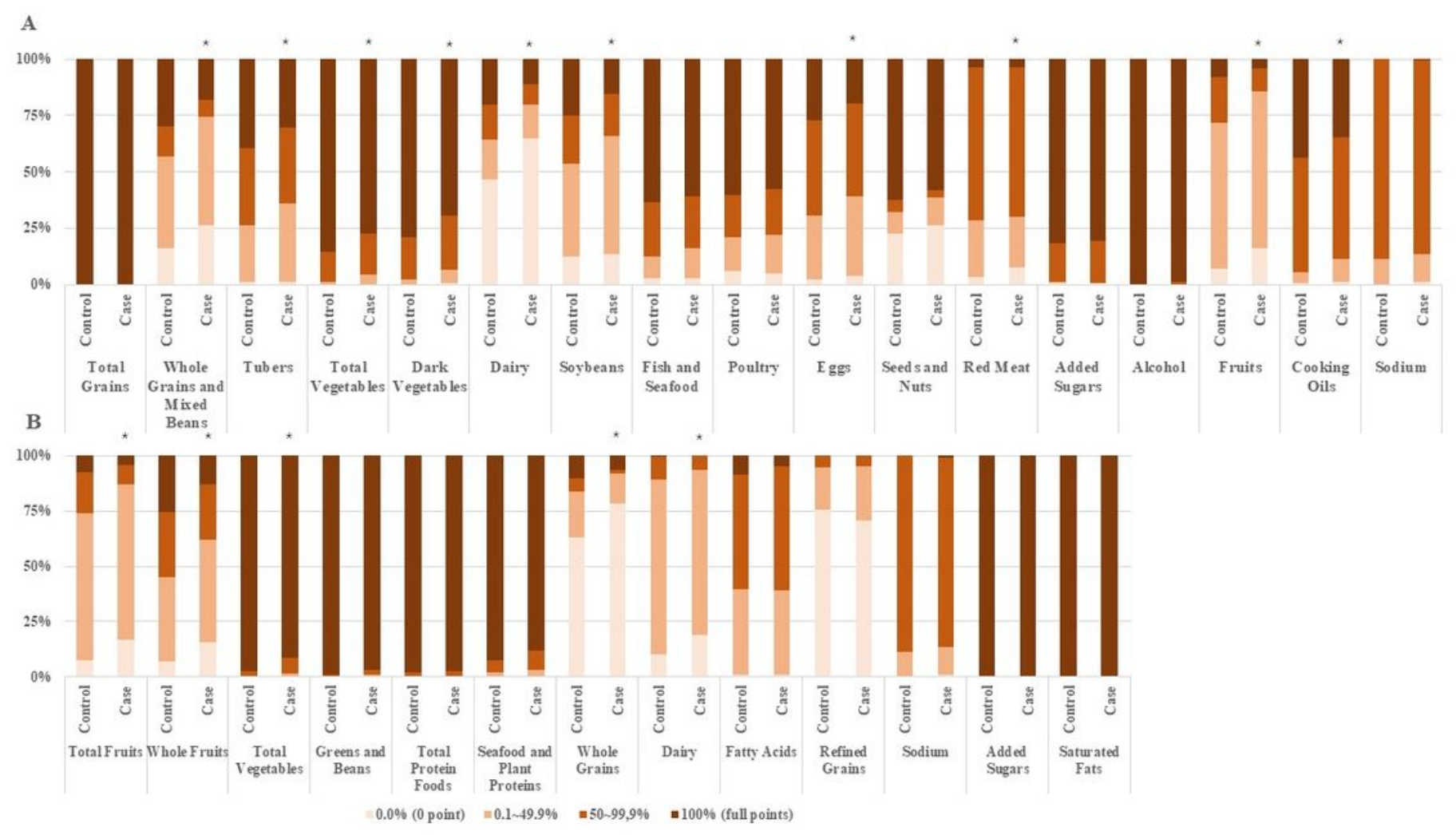

Figure 1

Comparison of the percentage distribution of the cases and controls according to the scores of each item for the $\mathrm{CHEI}(\mathrm{A})$ and the HEI-2015 (B). For fruits, sodium, and cooking oils in the CHEl and whole grains, dairy, fatty acids, refined grains, sodium, added sugars, and saturated fats in the HEI-2015, the cutoffs for the four groups are $0,0.1-4.9,5.0-9.9$, and 10.0. For the remaining components in the CHEl and the HEl2015 , the cutoffs for the four groups are $0,0.1-2.4,2.5-4.9$, and 5.0. *: $p<0.05$ (chi-square test). 


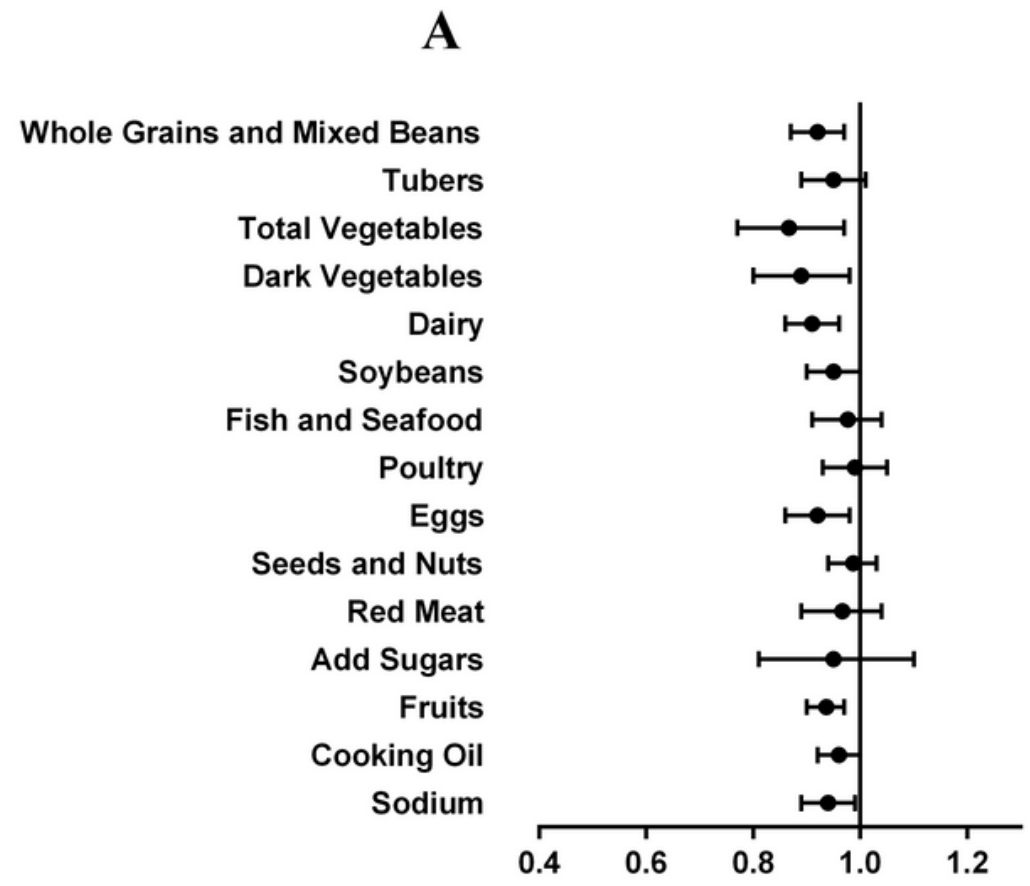

$\begin{array}{lc}\text { OR }(95 \% \text { CI }) & \boldsymbol{p} \text {-value } \\ \mathbf{0 . 9 2 ( 0 . 8 7 , 0 . 9 7 )} & \mathbf{0 . 0 0 1} \\ 0.95(0.89,1.01) & 0.121 \\ \mathbf{0 . 8 6}(\mathbf{0 . 7 7 , 0 . 9 7 )} & \mathbf{0 . 0 1 5} \\ \mathbf{0 . 8 9}(\mathbf{0 . 8 0 , 0 . 9 8 )} & \mathbf{0 . 0 1 9} \\ \mathbf{0 . 9 1}(\mathbf{0 . 8 6 , 0 . 9 6 )} & \mathbf{0 . 0 0 1} \\ 0.95(0.90,1.00) & 0.066 \\ 0.98(0.91,1.04) & 0.455 \\ 0.99(0.93,1.05) & 0.752 \\ \mathbf{0 . 8 6}(\mathbf{0 . 9 2 , 0 . 9 8}) & \mathbf{0 . 0 1 5} \\ 0.99(0.94,1.03) & 0.553 \\ 0.97(0.89,1.04) & 0.408 \\ 0.94(0.81,1.10) & 0.441 \\ \mathbf{0 . 9 4}(\mathbf{0 . 9 0 , 0 . 9 7 )} & \mathbf{0 . 0 0 1} \\ 0.96(0.92,1.00) & 0.078 \\ \mathbf{0 . 9 4 ( 0 . 8 9 , 0 . 9 9 )} & \mathbf{0 . 0 4 6}\end{array}$

\section{B}

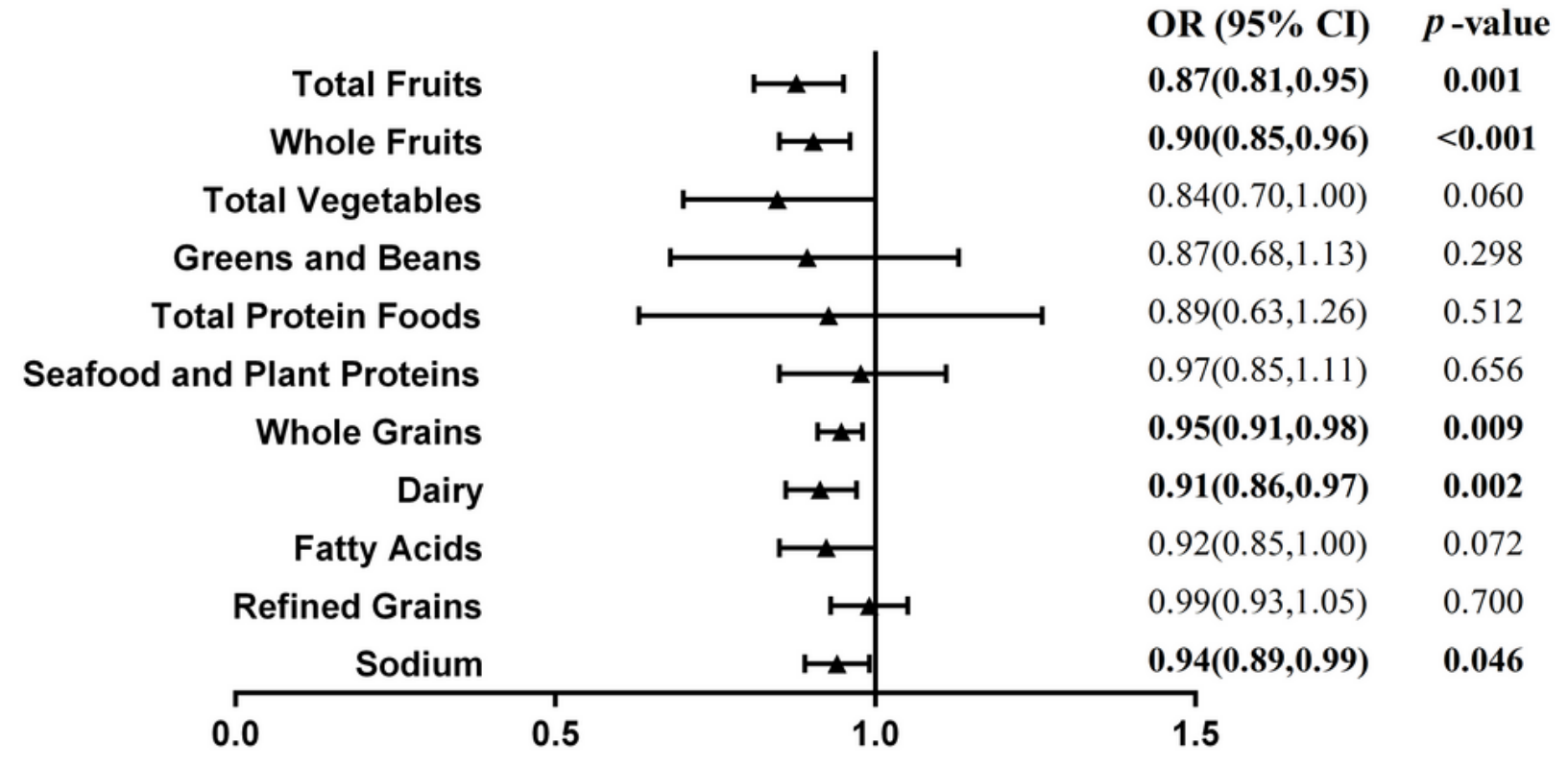

Figure 2

Association of each component score of CHEI (A) and HEI-2015 (B) with cardiovascular complications risk between case and control. ORs were adjusted for age, body mass index, marital status, physical activity, education level, smoker status, alcohol consumption, tea-drinking status, hypertension status, dyslipidemia status, T2D duration, and non-alcohol energy. Fatty acids, A ratio of total unsaturated fatty 
acids (poly- and monounsaturated fatty acids [PUFAs and MUFAs]) to saturated fatty acids (SFAs). Statistically significant results are bolded.

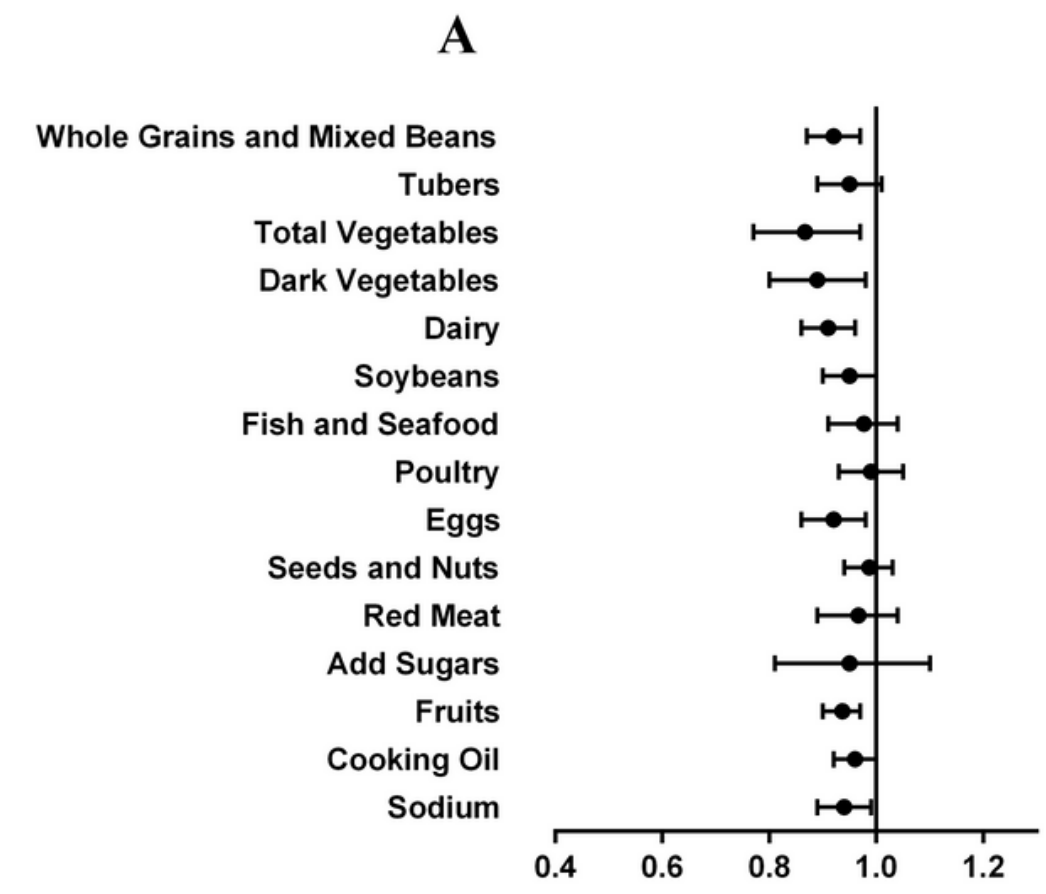

$\begin{array}{lc}\text { OR }(\mathbf{9 5 \%} \text { CI) } & \boldsymbol{p} \text {-value } \\ \mathbf{0 . 9 2 ( 0 . 8 7 , 0 . 9 7 )} & \mathbf{0 . 0 0 1} \\ 0.95(0.89,1.01) & 0.121 \\ \mathbf{0 . 8 6}(\mathbf{0 . 7 7 , 0 . 9 7 )} & \mathbf{0 . 0 1 5} \\ \mathbf{0 . 8 9 ( 0 . 8 0 , 0 . 9 8 )} & \mathbf{0 . 0 1 9} \\ \mathbf{0 . 9 1 ( 0 . 8 6 , 0 . 9 6 )} & \mathbf{0 . 0 0 1} \\ 0.95(0.90,1.00) & 0.066 \\ 0.98(0.91,1.04) & 0.455 \\ 0.99(0.93,1.05) & 0.752 \\ \mathbf{0 . 8 6}(\mathbf{0 . 9 2 , 0 . 9 8}) & \mathbf{0 . 0 1 5} \\ 0.99(0.94,1.03) & 0.553 \\ 0.97(0.89,1.04) & 0.408 \\ 0.94(0.81,1.10) & 0.441 \\ \mathbf{0 . 9 4}(\mathbf{0 . 9 0 , 0 . 9 7}) & \mathbf{0 . 0 0 1} \\ 0.96(0.92,1.00) & 0.078 \\ \mathbf{0 . 9 4}(\mathbf{0 . 8 9 , 0 . 9 9 )} & \mathbf{0 . 0 4 6}\end{array}$

B

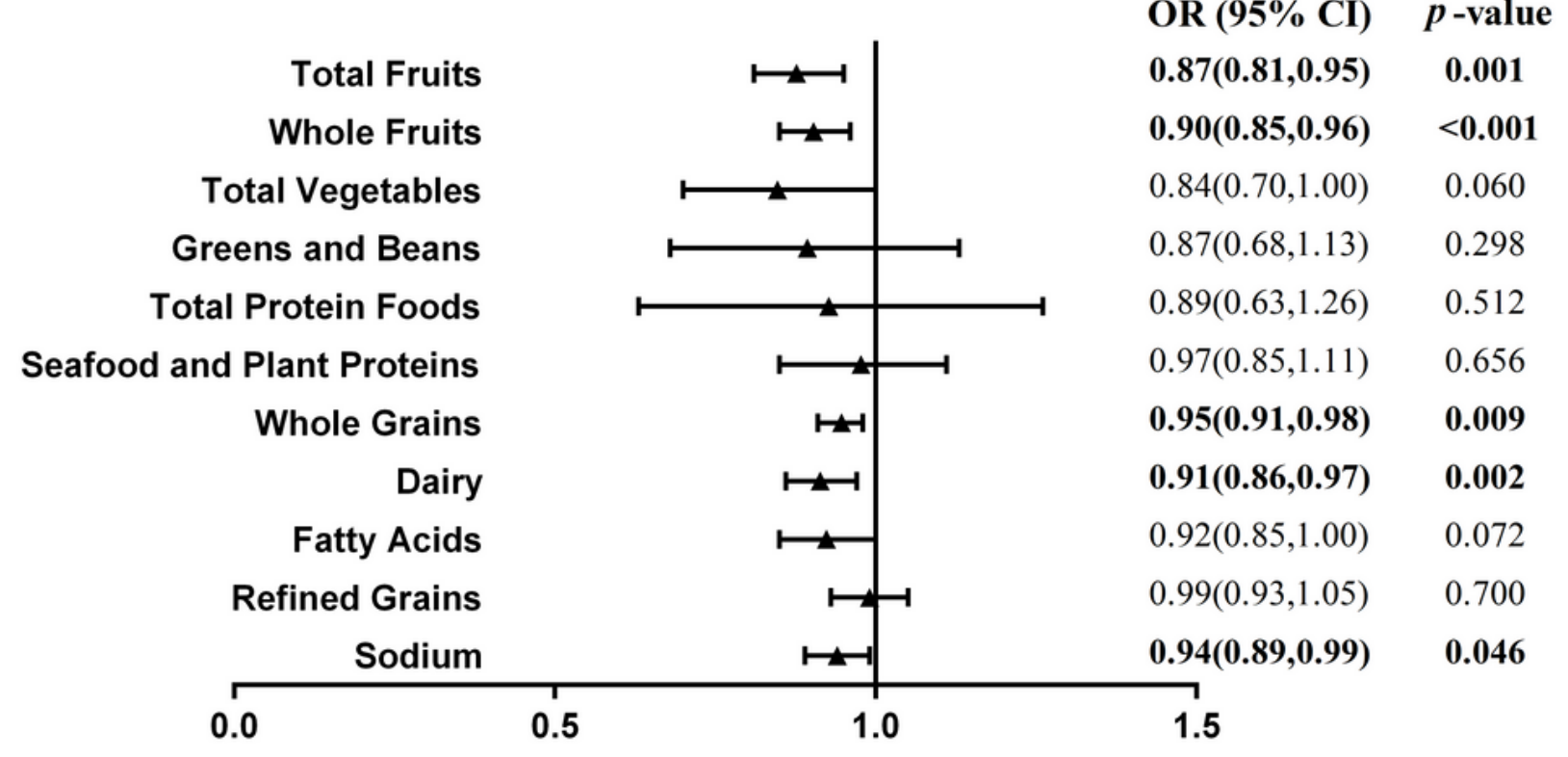

Figure 2

Association of each component score of $\mathrm{CHEI}$ (A) and HEl-2015 (B) with cardiovascular complications risk between case and control. ORs were adjusted for age, body mass index, marital status, physical activity, education level, smoker status, alcohol consumption, tea-drinking status, hypertension status, 
dyslipidemia status, T2D duration, and non-alcohol energy. Fatty acids, A ratio of total unsaturated fatty acids (poly- and monounsaturated fatty acids [PUFAs and MUFAs]) to saturated fatty acids (SFAs). Statistically significant results are bolded.

\section{Supplementary Files}

This is a list of supplementary files associated with this preprint. Click to download.

- SupplementaryMaterial.docx

- SupplementaryMaterial.docx 